\title{
Time-domain Impedance Boundary Conditions for Computational Acoustics and Aeroacoustics
}

\author{
K.-Y. FUNG ${ }^{\mathrm{a}, *}$ and HONGBIN JU ${ }^{\mathrm{b}, \dagger}$ \\ ${ }^{\mathrm{a}}$ Department of Mechanical Engineering, Hong Kong Polytechnic University, Kowloon, Hong Kong; ${ }^{\mathrm{b}}$ Department of Mathematics, \\ Florida State University, Tallahassee, FL 32306, USA
}

\begin{abstract}
This paper reviews the short history, motivation, numerical and theoretical issues, and development of methods for treating a boundary as a reflective/absorptive surface for the time-domain computation of waves in general and acoustic waves in particular. It begins with the extension and implementation of the frequency-domain impedance to a time-domain impedance-equivalent boundary condition (TDIBC), and illustrates how the theoretical, numerical, and implementation issues are addressed and resolved for acoustic/aeroacoustic applications. Comments are also made on the extendibility and applicability of the concept and TDIBC to other fields and types of problems.
\end{abstract}

Keywords: Computational aeroacoustics; Impedance boundary condition; Time-domain method

\section{INTRODUCTION}

The classical approach to the solution of a space-timevarying problem $\phi(\vec{x}, t)$ is the conversion of the differential/integral time dependency to a parametric frequency dependency $\omega$ through the Fourier transform, or the assumption of the time harmonic behavior: $\hat{\phi}(\vec{x}, \omega) e^{i \omega t}$. This conversion reduces the space-time initial-boundary value problem to a boundary value problem with the parametric dependency $\omega$. System linearity is assumed, and the admissible conditions on some boundary $\Gamma$ can be cast as a ratio between a transformed dependent variable $\hat{\phi}(\vec{x}, \omega)$ and its normal derivative $\hat{\phi}_{n}$ in the form of an impedance $Z(\omega)$. For example, in the transformed frequency-space domain $D$ the wave equation of wave speed $C_{0}$ becomes the spatially second-order Helmholtz equation $\nabla^{2} \hat{\phi}+\left(\omega / C_{0}\right)^{2} \hat{\phi}=0$ of the elliptic type. To render a solution of this equation on a domain $D$, a constraint in the general form $a \hat{\phi}+b \hat{\phi}_{n}=0$ on the contour $\Gamma$ bounding $D$ in all directions is necessary. Here, $a$ and $b$ are complex functions of $\omega$, and the subscript $n$ denotes the spatial derivative normal to $\Gamma$. Since the $k$ th-order temporal derivative of $\phi$ is transformed into $(i \omega)^{k} \hat{\phi}$, in particular $p=-\phi_{t}=-i \omega \hat{\phi}$ with sound pressure $p$ and sound velocity potential $\phi$ in acoustics, this general constraint can be viewed as the ratio of $\hat{p} / \hat{\phi}_{n}$, which in the transformed space is simply $i \omega b / a=Z(\omega)$. The earliest uses of the term impedance date back to O. Heavyside's 1886 description in circuit theory of "the ratio of the impressed force to the current in a line", and to A.G. Webster's 1914 description in acoustics of the ratio between harmonic components of the pressure $p$ and fluid velocity $u_{n}$ into a porous wall (Pierce, 1991, pp. 107-108). Customarily, impedance is expressed in the complex form $Z=R+i X$ of real functions $R(\Gamma, \omega)$ and $X(\Gamma, \omega)$, respectively the resistance and reactance (inductance in electromagnetism), where $i^{2}=-1$. In the literature, where time harmonic behavior $e^{-i \omega t}$ is assumed the sign of $X$ in $Z$ is negative $(Z=R-i X)$. Impedance, often a measured quantity in practice, is a means to characterize the absorption and reflection of waves at a surface. It is locally reactive if impedance is just a property of the surface and independent of incident wave angle $\theta$. The variation of $Z(\Gamma, \omega)$ along the boundary $\Gamma$ is assumed, taking values in the right half complex $Z$-plane, $R \in[0, \infty)$ and $X \in(-\infty, \infty)$. Figure 1 shows some measured resistance and reactance values, normalized by air impedance $\rho_{0} C_{0}$, of a typical perforated treatment panel for aeroacoustic applications. These values give for each frequency a simple algebraic closure to the boundary value problem of the mixed Neumann-Dirichlet type, for which numerous analytic/numerical solution techniques have been proposed.

The rapid and successful development of computational methods since the advent of the modern computer in the late 1960s has popularized their use as part of the routines

*Corresponding author. Tel.: + 852-2766 6644. Fax: +852-2365-4703. E-mail: mmkyfung@polyu.edu.hk

'Tel.: + 1-850-6450185. E-mail: hju@ math.fsu.edu 


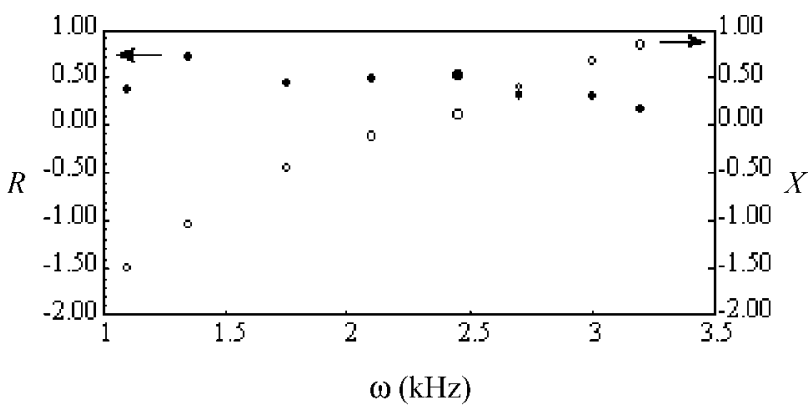

FIGURE 1 Normalized resistance $R$ (solid dots) and reactance $X$ (open dots) of a perforated treatment panel for sound absorption.

in design, and spurs further demands for more efficient algorithms and the inclusion of increasingly more complex and multidimensional models. These methods have advanced from simple geometry, single dimension, linear, time-stationary systems to complex geometry, multi-dimension, nonlinear, time-progressing systems as rapidly as the size and speed of the computer allow. The quest for more efficient algorithms, better discretization of a given finite or infinite space and faster attainment in real time of an accurate solution continues, nonetheless. Higher-order algorithms, in the context of either finite difference or finite element methods, are still being pursued and are believed to correspond to smaller CPU sizes, more accurate solution representation, and faster solution processes. However, the rapid attainment of a time-stationary solution, or the accurate prediction of a time-progressing one, depends on the way these algorithms are closed and the type of specified value at domain boundaries. The progression of an initialboundary-value problem from one state to another or eventually to a steady state can be viewed as propagation of waves, or spreading of locally unsupported nonuniformity in an equivalent wave-like process. The effect of a closure, numerical or physical, to the progression of the wave-like features in a solution towards a boundary can be viewed as reflection from an impedance surface. Characterization and design of conditions for closure of a boundary should be of great interest to the developers of numerical methods.

In computational aeroacoustics (CAA), time-domain methods have great advantages over frequency-domain methods for broadband problems, non-linear interactions investigation and transient wave simulations. The use of impedance in the context of a time-domain computation first appeared separately in electromagnetism and acoustics communities. Maloney and Smith (1990) proposed the replacement of the region of lossy dielectric by the impedance of the interface, so to reduce the size of the grid in the finite-difference time-domain (FD-TD) solution of electromagnetic (EM) wave in a free space bounded by a lossy dielectric half space. The analytic impedance $Z(\omega)$ in this case can be expressed and transformed into the kernel $\bar{Z}(t)$ of a time-convolution between the tangential components of the electric field $E$ and curl of the magnetic field $H$ at the interface.
By an application of Prony's method, the time-decaying kernel is approximated in a time-exponential series $\bar{Z}(t) \approx \sum_{k} \mu_{k} e^{-\Omega_{k} t}$ to allow the evaluation of the otherwise time-consuming convolution integral by a recursive formula. Later, Maloney and Smith (1992) demonstrated the FD-TD computation of the reflection of plane wave by a lossy dielectric model of $Z(\omega)$ and the confinement of an EM wave in parallel walls with an impedance discontinuity. Beggs et al. (1992) proposed for constant surface impedance direct operational conversion of $i \omega$ into the time derivative $\partial / \partial t$, and for broadband impedance fitted time-exponential series for efficient recursive integration. They presented the reflection of a Gaussian pulse in one dimension and the scattering by a conducting cylinder in two dimensions. Yee et al. (1992) recognized the requirement of a positive inductive impedance, $X\left(\Omega_{0}\right)>0$, for algorithmic stability, and proposed the alternative conversion of the admittance $Y\left(\Omega_{0}\right)=1 / Z\left(\Omega_{0}\right)=(R-$ $i X) /\left(R^{2}+X^{2}\right)$ to FD-TD operators when $X\left(\Omega_{0}\right)<0$. They demonstrated their surface impedance/admittance schemes on the FD-TD computation of the scattering of EM waves by an ellipsoid represented on a rectangular grid. Lee et al. (1992) proposed a rational expansion in $\omega$ of the two-layer-coated-conductor impedance model for operational conversion into a time-domain impedance condition. They presented the FD-TD computation of the reflection of Gaussian pulse in one dimension. Sullivan (1992) proposed the use of $z$-transforms for the development of frequency-dependent FD-TD methods for complex EM systems. He demonstrated the computation of plane EM-wave propagation in layered dielectric media. Oh and Schutt-Aine (1995) proposed an expansion of the impedance model of a lossy dielectric in the Laplace-plane by a rational Chebyshev approximation, which corresponds to a series of time-decaying exponential functions that can be tabulated and referenced for FD-TD implementation. Yee and Chen (1997) extended the method of Yee et al. (1992) to an unstructured finite-volume time-domain formulation and demonstrated the scattering of EM waves by spherical and ellipsoidal objects of constant surface impedance. Roden and Gedney (1999) pointed out that the manner in which Prony's method was employed by Maloney and Smith (1992) and Beggs et al. (1992) for fitting the convolution kernel with time-decaying exponential series might not converge correctly as finer time-steps $\Delta t$ were taken, but that polynomial-fitting a specific application range for partial-fraction representation of $Z$ in the Laplace plane $(s=i \omega)$ not only allowed correct convergence as finer steps $\Delta t$ were taken, but also improved the representation of $\mathrm{Oh}$ and Schutt-Aine (1995). They then proposed a way to implement the impedance boundary condition in generalized curvilinear coordinates and benchmarked their schemes on parallel and coaxial wave-guides.

In acoustics, Davis (1991) reported the incorporation of a low-frequency impedance model of the open pipe end by 
direct operational conversion of powers of $i \omega$ to time derivatives of $\partial / \partial t$. He demonstrated the direct timedomain computation of multiple reflections of acoustic impulse in a pipe. Botteldooren (1994) studied the accuracy of a second-order leapfrog scheme when used on quasi-uniform rectangular grids closed with a simple time-equivalent impedance model of the damped harmonic oscillator. He used the combined scheme to simulate lowfrequency room acoustics (Botteldooren, 1995). Neither Davis nor Botteldooren offered any detail or discussed any problem in their implementation of the impedance boundary conditions. Tam and Auriault (1996) first addressed the instability problem in the implementation of TDIBC, and demonstrated the construction of stable impedance-dependent boundary schemes for reflection of harmonic waves and banded pulses in one dimension. Their schemes were recently applied and verified on multidimensional acoustic problems by Zheng and Zhuang (2002). Tam and Auriault (1996) also explored the construction of a convection-modified impedance condition, and proposed the use of impedance measured under flow condition to avoid instability of the KelvinHelmholtz type found in their analysis. Özyörük and Long (1997) proposed a rational representation of measured impedance and conversion of time-domain impedance operators via the $z$-transform. They demonstrated their method on the reflection of Gaussian pulse in one and two dimensions and on the absorption of waves in a flow duct with a partially lined impedance wall (Özyörük et al., 1998, 2000). Fung and TallaPragada (1997) pointed out an inherent instability in the operational conversion of an expansion of impedance to time-domain differential operators and proposed instead the conversion of reflection coefficient $W(\omega)$ defined as the bilinear transform $(1-Z) /(1+Z)$. Subsequently, Fung et al. (2000) proposed stable, $\hat{W}(\omega)$-based, differential and integral TDIBC formulations and benchmarked their methods on the reflection of impulses in one dimension and on an analytically constructed initial-impedance-boundaryvalue solution of impulse reflection in two dimensions. Their methods have since been extended to duct acoustics in three dimensions by Ju and Fung (2000), acoustics in partially lined flow ducts in two dimensions (2001), and reflection of acoustic sources by an impedance plane for outdoor acoustics (2002).

In the following we will delineate and compare methods for the construction and implementation of TDIBC for wave propagation in wall-bounded, stationary or convective media, and draw on the importance of causality in bridging frequency- and time-domain impedance properties.

\section{CAUSAL IMPEDANCE}

Some theoretic background is needed to facilitate a better understanding of the various methods proposed for TDIBC construction. Let us begin with the familiar definition of impedance:

$$
\hat{p}(\omega)=Z(\omega) \hat{u}(\omega)
$$

where $\hat{p}(\omega)$ and $\hat{u}(\omega)$ are harmonic components with time factor $e^{i \omega t}$, or Fourier-transformed variables, of the perturbed pressure $p(t)$ and its induced normal velocity component $u(t)$ into a porous surface $\Gamma$, and assume for simplicity that $Z(\omega)$ is locally reactive. Here, circular frequency $\omega$ has been normalized by sound speed $C_{0}$ and characteristic length $L ; \hat{p}$ by $\rho_{0} C_{0}^{2}$ with mean air density $\rho_{0}, \hat{u}$ by $C_{0}$, and $Z$ by $\rho_{0} C_{0}$. Readers are reminded here of the equivalence of Laplace and Fourier transforms by the simple replacement of $s=i \omega$. Equation (1) is often considered a linear system of input $\hat{u}(\omega)$, response function $Z(\omega)$ and output $\hat{p}(\omega)$. It has the equivalent time convolution of

$$
p(t)=\int_{-\infty}^{\infty} \bar{Z}(\tau) u(t-\tau) \mathrm{d} \tau
$$

where $\bar{Z}(t)$ is the inverse Fourier transform of $Z(\omega)$. By Cauchy's theorem, unless all poles $\lambda_{k}$ of $Z(\omega)$ lie in the upper complex $\omega$-plane, i.e. $\operatorname{Img}\left(\lambda_{k}\right)>0$, the current value of $p(t)$ would involve future values of $u(t)$ in $\int_{-\infty}^{0} \bar{Z}(\tau) u(t-\tau) \mathrm{d} \tau$, which of course is unphysical for the violation of causality. If causality, a property of the impedance model $Z(\omega)$, is violated but somehow implemented without a deliberated effort, instability would result. Causality and thus stability depend on how $Z(\omega)$, often in practice a measured quantity, is analytically represented. The use of time-exponential series in the formulations of Luebbers et al. (1990), Maloney and Smith (1992), Oh and Schutt-Aine (1995) and Roden and Gedney (1999) is basically an observation of causality.

Mathematically, Eq. (1) can also be rewritten as:

$$
\hat{u}(\omega)=Y(\omega) \hat{p}(\omega) .
$$

Here, $Y(\omega)=1 / Z(\omega)$, called the acoustic admittance, reverses the input and output relation in Eq. (1). Hence, all poles of $Y(\omega)$, or zeros of $Z(\omega)$, must lie in the upper complex $\omega$-plane for Eq. (3) to be causal in time domain. If $Z(\omega)$ is expressed in a rational form $A(\omega) / B(\omega)$, as proposed in Oh and Schutt-Aine (1995) and Özyörük and Long (1997), the zeros of $B(\omega)$ and $A(\omega)$ must lie in the upper $\omega$-plane, respectively for Eq. (1) and Eq. (3).

To understand further the time-equivalent process, let us inspect a simple fixed-point impedance value, i.e. $Z=R_{0}+i X_{1}$ at $\omega=\Omega_{0}$, and a consistent broadband model: $Z(\omega)=R_{0}+i X_{1} \omega / \Omega_{0}$. This model has no pole and only one zero at $\omega=i \Omega_{0} R_{0} / X_{1}$. Either $\Omega_{0} R_{0} / X_{1}>0$ or the equivalent time process would violate causality and lead to unstable schemes, as found in Yee et al. (1992) and in Tam and Auriault (1996). While positive resistance $\left(R_{0}>0\right)$ is a physical mandate, reactance may assume values of either sign. In this form, only positive $X_{1}$ corresponds to stable TDIBC. Fortunately, there is 
an alternative to this fixed-point model. Consider instead $Z(\omega)=R_{0}+i X_{1} \Omega_{0} / \omega$, which has the same impedance value at $\Omega_{0}$, a pole at $\omega=0$ and a zero at $\omega=-i X_{1} \Omega_{0} / R_{0}$, effectively flipping the zero to the upper complex $\omega$-plane for negative $X_{1}$. This is the remedy suggested in Tam and Auriault (1996). The practicality of finding alternative broadband causal TDIBC models in such a way is doubtful. As pointed out in Fung et al. (2000), the use of a higher-order model in $\omega$, e.g. $Z(\omega)=R_{0}+R_{2} \omega^{2}+\mathrm{i} X_{1} \omega$, would likely lead to roots of opposite signs, thus always in violation of causality.

It helps at this point to compare TDIBC implementations on the simple broadband model:

$$
Z(\omega)=R_{0}+i X_{1} \omega
$$

which corresponds to:

$$
p(t)=R_{0} u(t)+X_{1} \frac{\mathrm{d} u}{\mathrm{~d} t}
$$

or

$$
u(t)=\frac{1}{X_{1}} \int_{0}^{\infty} e^{-\frac{R_{0}}{X_{1}} \tau} p(t-\tau) \mathrm{d} \tau
$$

The differential formulation Eq. (5a) when discretized by two-level finite differences may assume the following form:

$$
\begin{aligned}
u^{n}= & \frac{[1-(1-\vartheta) \kappa]}{1+\vartheta \kappa} u^{n-1}+\frac{\Delta t}{X_{1}(1+\vartheta \kappa)} \\
& \times\left[\vartheta p^{n}+(1-\vartheta) p^{n-1}\right] .
\end{aligned}
$$

Here $\kappa=R_{0} \Delta t / X_{1}, \quad t=n \Delta t, \quad u^{n}=u(n \Delta t), \quad \tilde{t}=(n-$ $1+\vartheta) \Delta t, \quad \vartheta \in[0,1]$ is the weighting factor in the weighted quadrature $\int_{t-\Delta t}^{t} p(t) \mathrm{d} t \approx\left[\vartheta p^{n}+(1-\right.$ ๆ) $\left.p^{n-1}\right] \Delta t$, and $p(t)$ as the input is assumed known. Equation (6a) is recursive in $u^{n}$ with the amplification factor $z_{\Delta}=[1-(1-\vartheta) \kappa] /(1+\vartheta \kappa)$, and conditionally stable for $\left|z_{\Delta}\right|<1$; or $0<\kappa<2 /(1-2 \vartheta)$ for $\vartheta<1 / 2$ and $0<\kappa$ for $\vartheta>1 / 2$. Equation (6a) shows that if causality $R_{0} / X_{1}>0$ is not observed algorithmic instability results even with the fully implicit method of $\vartheta=1$.

By relating $u(t), u(t-\Delta t)$ and $z=e^{-\kappa}$, Eq. (5b) can be approximated recursively as:

$$
\begin{aligned}
u^{n} & =z u^{n-1}+\frac{1}{X_{1}} \int_{0}^{\Delta t} e^{-\frac{R_{0}}{X_{1}} \tau} p(t-\tau) \mathrm{d} \tau \\
& \approx z u^{n-1}+\frac{\Delta t}{X_{1}}\left[\vartheta p^{n}+(1-\vartheta) z p^{n-1}\right] .
\end{aligned}
$$

The advantages of the integral formulation for TDIBC are seen in Eq. (6b), which is unconditionally stable for all admissible parametric ranges and second-order accurate in time when $\vartheta=1 / 2$. Whereas the approximation of $z=e^{-\kappa}$ by various two-level difference operators $z_{\Delta}$ results in only conditionally stable time-domain formulas and a poor quadrature for Eq. (5b) for large $\kappa$, cf. Eqs. (6a) and (6b). This suggests that unless the poles $\lambda_{k}$ of $Z(\omega)$ or $Y(\omega)$ are identified in the complex frequency plane and used explicitly for time advancement, an operational approximation such as the rational form $Z=A\left(z_{\Delta}\right) / B\left(z_{\Delta}\right)$ proposed in Özyörük and Long (1997) and Özyörük et al. (1998) would lead to conditional stable schemes at best.

\section{SPACE-TIME CONTINUATION}

So far, we have been working with loosely interchangeable time sequences of input and output and a sense of causality given by the response function $\bar{Z}(t)$ or $\bar{Y}(t)$. We now look at a physical system of wave propagation in the domain $x \in\left(x_{a}, x_{b}\right)$ governed by, e.g. the wave equation $\partial^{2} \phi / \partial t^{2}-C_{0}^{2} \partial^{2} \phi / \partial x^{2}=0$. There is no loss of generality to set from here on the wave speed $C_{0}=1$ or rescale $x$ and $t$ appropriately to absorb this dimensionality. The general solution of the rescaled space-time hyperbolic system comprises a left-running wave $u^{-}(x+t)$ and a rightrunning wave $u^{+}(x-t)$ along their respective left- and right-running characteristics $x+t$ and $x-t$. These waves, the characteristic variables, are related to the physical variables, pressure $p(x, t)=-\partial \phi / \partial t$ and particle velocity $u(x, t)=\partial \phi / \partial x$, by $u^{+}(x-t)=u(x, t)+p(x, t)$ and $\quad u^{-}(x+t)=u(x, t)-p(x, t) ; \quad$ or conversely, $u=\left(u^{+}+u^{-}\right) / 2$ and $p=\left(u^{+}-u^{-}\right) / 2$. Both waves would be independent if it were not for the boundary conditions to be imposed at $x_{a}$ and $x_{b}$ on $u, p$, or a general constraint as Eq. (2). Along its characteristic $x-t$ the right-running wave $u^{+}(x, t)$ at a space-time point $(x, t)$ is related to the value $u^{+}(x-\Delta t, t-\Delta t)$ at the point $x-\Delta t$ to the left and the earlier time $t-\Delta t$. This means that all values of $u^{+}(x, t)$ found at $x$ are related to earlier values at points to the left of $x$, values that had existed in the past and therefore cannot be changed or subjected to any future constraint. In particular, the rightmost value of the rightrunning wave $u^{+}\left(x_{b}, t\right)$ at the boundary point $x_{b}$ cannot be specified or constrained, such as Eq. (1), nor can the left running wave $u^{-}\left(x_{a}, t\right)$ at the left boundary point $x_{a}$. To satisfy a constraint, e.g. the hard wall condition of $u=\left(u^{+}+u^{-}\right) / 2=0, \quad$ the domain-entering wave (the reflection) must be determined from the domainexiting wave (the incident) so not to violate space-time causality. Therefore, $u^{-}\left(x_{b}, t\right)$ is set to equal $-u^{+}\left(x_{b}, t\right)$ to maintain $u\left(x_{b}, t\right)=0$, and $u^{-}\left(x_{a}, t\right)$ equal $-u^{+}\left(x_{a}, t\right)$ to maintain $u\left(x_{a}, t\right)=0$. In other words, the reflection is the space-time continuation of the incident with reference to a general wall condition, such as Eq. (2).

The imposition of a constraint on $\hat{p}(x, \omega)$ or $\hat{u}(x, \omega)$ in the space-frequency plane, in which the corresponding Helmholtz equation is elliptic, does not need to observe a similar causality requirement. In fact, no such 
requirement is allowed for elliptic systems. The harmonic assumption already implies that $u(x, t)$ and $p(x, t)$ are of the form $e^{i \omega t}$ and available for $-\infty<t<\infty$. The closure for $\hat{p}(x, \omega)$ or $\hat{u}(x, \omega)$ in the form of Eq. (1) is fully admissible without the requirement of a causal $Z(\omega)$ for their relation.

The principal difference between a frequencydomain approach and a time-domain approach is the need to observe causality, without which a constraint such as Eq. (2), the time-domain equivalent of Eq. (1), is simply not enforceable in the time domain. Which then should be implemented, Eq. (1) or Eq. (3), for a time-domain solution of the wave equation? Or, what form of $Z(\omega)$ should be used for the construction of its timedomain equivalent?

Suppose Eq. (4) is to be imposed on $u\left(x_{b}, t\right)$ at $x_{b}$ where the incident $u^{+}\left(x_{b}, t\right)$ is known and for which the relation between $u$ and $p$ in Eq. (5a) is replaced by the relation between $u$ and $u^{+}\left(x_{b}, t\right)$, i.e. $p^{n}=u^{+}-u^{n}$. The corresponding recursive formula for $u^{n}$ has the reduced stability of $0<\kappa^{\prime}=\left(R_{0}+1\right) \Delta t / X_{1}<2 /(1-2 \vartheta)$ for $\vartheta<1 / 2$ and $0<\kappa^{\prime}$ for $\vartheta>1 / 2$. This reduction of stability is due to the fact that part of $u^{n}$ is already causally given in the incident wave, $u^{+}=u+p$, and its future values would be overly predicted if $\Delta t$ was not correspondingly reduced. Similar replacement in Eq. (6b) results in the modified conditional stability of $\Delta t / X_{1}<(1+z) /[z-\vartheta(1+z)]$ for $\vartheta<z /(1+z)$. Here the unconditional stability of Eq. (6b) is reduced to a conditional one for the zero of $Z$ was used to form the recursive factor $z$ rather than the zero of $1+Z$ in the modified impedance of $u^{+}=\hat{p}+\hat{u}=(1+Z) \hat{u}$.

Neither $u$ nor $p$ is characteristically independent of the incident $u^{+}$at $x_{b}$ and a suitable choice for the enforcement of a time-domain equivalent of Eq. (1) for the wave equation. It is the left-running wave $u^{-}$at a right boundary that is characteristically independent of the incident $u^{+}\left(x_{b}, t\right)$ and, therefore, the most suitable candidate for its causal space-time continuation. Indeed, such a relation is given by the reflection coefficient $\hat{W}(\omega)$,

$$
\hat{u}^{-}(\omega)=\hat{W}(\omega) \hat{u}^{+}(\omega)
$$

where $\hat{W}(\omega) \equiv(1-Z) /(1+Z)$. By its very name the complex-valued coefficient gives a measure of the frequency-dependent magnitude and phase angle of the reflection with respect to the incident. It maps bilinearly the entire right $Z$-plane of physically admissible impedance, Real $(Z)>0$, into the interior of the unit circle $|\hat{W}|<1$ in the $\hat{W}$-plane. Typical values of $\hat{W}(\omega)$ are 0 for no reflection, -1 for total reflection from a hard surface of $u=0$, and +1 from a pressure surface of $p=0$. It can be succinctly expressed as a measure of wall softness, $\hat{\tilde{W}}(\omega) \equiv 2 /(1+Z)=1+\hat{W}(\omega)$, from the hard reflection condition of $\hat{W}(\omega)=-1$. As $|\hat{W}|<1$ the amplification of $\hat{u}^{+}$is bounded by unity. The requirement that all poles $\lambda_{k}$ of $\hat{W}(\omega)$, or zeros of $1+Z(\omega)$, be on the upper half $\omega$-plane further ensures that reflection is causally convoluted from incident:

$$
u^{-}(t)=-u^{+}(t)+\int_{0}^{\infty} \tilde{W}(\tau) u^{+}(t-\tau) \mathrm{d} \tau .
$$

Here $\tilde{W}(\tau)=H(\tau) \sum_{k} \mu_{k} e^{i \lambda_{k} \tau}, \mu_{k}=i \cdot \operatorname{Residue}\left[\hat{\tilde{W}}(\omega), \lambda_{k}\right]$, and $H(t)$ is the Heavyside's step function which switches on the exponentially decaying $e^{i \lambda_{k} \tau}$ for each of the causal poles, $\operatorname{Img}\left(\lambda_{k}\right)>0$ of $1+Z$. Equation (8) implemented in the same way as Eq. (6b), with $z_{k}=e^{i \lambda_{k} \Delta t}$, has the form:

$$
\begin{gathered}
u^{-}(t)=-u^{+}(t)+\sum_{k} u_{k}^{-}(t), \text { where } \\
u_{k}^{-}(t)=z_{k} u_{k}^{-}(t-\Delta t)+\mu_{k} \int_{0}^{\Delta t} e^{i \lambda_{k} \tau} u_{k}^{+}(t-\tau) \mathrm{d} \tau .
\end{gathered}
$$

Stability is assured since each subsystem $u_{k}^{-}(t)$ is stable with $\left|z_{k}\right|<1$.

This is the TDIBC system that Fung et al. (2000) advocated. It takes into account the space-time continuation of incident and reflected waves. It is unconditionally stable when the complex roots of $1+Z(\omega)=0$ are known and causal, and is characteristically proper to render a boundary closure of the wave equation on a domain bounded by $\Gamma$ by the provision of the domain-entering reflection $u^{-}(\Gamma, t)$.

Equation (9) can be easily approximated by quadratures of any order, e.g. the first-order backward Euler:

$$
u_{k}^{-}(t) \approx z_{k} u_{k}^{-}(t-\Delta t)+\Delta t \mu_{k} u_{k}^{+}(t),
$$

or the second-order trapezoidal rule:

$$
u_{k}^{-}(t) \approx z_{k} u_{k}^{-}(t-\Delta t)+\frac{1}{2} \Delta t \mu_{k}\left[u_{k}^{+}(t)+z_{k} u_{k}^{+}(t-\Delta t)\right]
$$

In most cases, the grid system and time step chosen to resolve $u^{+}(t)$ and $u^{-}(t)$ on Eq. (11) are sufficiently accurate. For very large $\left|\lambda_{k}\right|$, exact integration of $e^{i \lambda_{k} \tau}$ assuming a linearly varying $u^{+}(t)$ in Eq. (9) gives a substantially improved weighted quadrature at no extra computational cost:

$$
\int_{0}^{\Delta t} e^{i \lambda_{k} \tau} u^{+}(t-\tau) \mathrm{d} \tau \approx\left[w_{k 0} u^{+}(t)+w_{k 1} u^{+}(t-\Delta t)\right] \Delta t,
$$

where

$$
w_{k 0}=-\frac{z_{k}-1}{\lambda_{k}^{2} \Delta t^{2}}-\frac{1}{i \lambda_{k} \Delta t}
$$

and

$$
w_{k 1}=\frac{z_{k}-1}{\lambda_{k}^{2} \Delta t^{2}}+\frac{z_{k}}{i \lambda_{k} \Delta t} .
$$




\section{TDIBC MODELS}

We have come to realize that the construction of TDIBC hinges on finding the roots of $1+Z(\omega)=0$. Unfortunately, the identification of the complex roots when only discrete values of $Z(\omega)$ are known or defined on real $\omega$ is not routine or even mathematically unique. When the exact impedance impulse $\bar{Z}(t)$ is analytically known, Beggs et al. (1992) and Maloney and Smith (1992) proposed the use of Prony's method to obtain the approximate exponential representation $\bar{Z}(t)=\sum_{k} \mu_{k} e^{-\Omega_{k} t}$, and thus reduce it to a recursive formula. Oh and Schutt-Aine (1995) proposed the tabulation and use of rational Chebyshev polynomials, instead of Prony's method, to approximate the purely real analytic impedance of lossy dielectric interfaces on the real axis of the Laplace s-plane. Roden and Gedney (1999), however, pointed out that Prony's expansion on a finite time-interval might be inadequate for representing the decay of long time scales (small $\Omega_{k}$ ) and result in larger errors as smaller time steps $\Delta t$ were used. They suggested using the poles from polynomial-fitting a set of real $Z$ values on the real $s$-axis over an adequate application range.

In practice, only sets of discrete impedance values are measured and known on the real $\omega$-axis. Commercial software packages (Matlab, Mathematica, etc.) are readily available for fitting and numerically identifying the complex zeros, but the zeros so identified may not necessarily be causal, depending on the range and amount of data available. The choices of a model would also depend on the type of application. We now review these choices.

The most straightforward representation is a polynomial representation of impedance in the form:

$$
\begin{aligned}
Z(\omega) & =R(\omega)+i X(\omega) \\
& =R_{0}+R_{2} \omega^{2}+\cdots+i\left(X_{1} \omega+X_{3} \omega^{3}+\cdots\right) .
\end{aligned}
$$

This equation must be valid for some non-zero radius of convergence or its validity to have a corresponding time-equivalent process is doubtful. The reason for the even and odd expansions, respectively for $R$ and $X$, is to result in purely real time operators, as only even powers of $\omega$ and odd powers of $i \omega$ correspond to real timederivatives. As pointed out by Fung et al. (2000), Taylor's expansions in $\omega$ of orders higher than one are likely to encounter non-causal roots and lead to algorithmic instability. Therefore, Eq. (13) is limited to narrow-band applications. The bandwidth of waves present in a time-domain solution depends not only on the input signal forced or initiated in space, but also on the order of schemes and smoothness of solution. Highorder schemes often support high-order spurious waves and require a high degree of solution smoothness, which may be difficult to manage in a multi-dimensional problem. An impedance discontinuity in the boundary for example often incites the development of spurious waves, but an accurate enforcement of TDIBC would leave little room for the design of artificial damping for the suppression of spurious waves. Once present, the spurious waves having a much wider spectrum than the intended input waves would be amplified by the implemented TDIBC. This amplification would also extend to any finite Taylor's expansion of the reflection coefficient $\hat{W}(\omega)$, which may not be bounded despite the absence of poles in the expansion of $\hat{W}(\omega)$.

Only the integral approach of Eq. (8), which necessitates the identification of the zeros of $1+Z(\omega)=0$, would provide the broadband boundedness. We should therefore focus on models of softness coefficient $\hat{\tilde{W}}(\omega)=2 /(1+Z)$ in the form $N(s) / D(s)$ where $N(s)$ and $D(s)$ are factored polynomials, i.e. $D(s)=\left(s-\lambda_{l}\right)\left(s-\lambda_{2}\right) \cdots\left(s-\lambda_{m}\right)$. The replacement of $i \omega$ by $s$ is a preference over the zeros of $D(s)$ on the real $s$-axis of the Laplace-plane. Thus, $\tilde{W}(\omega)$ may be expanded in the partial-fraction form: $\tilde{W}(\omega)=$ $\sum_{k=1}^{m} \hat{\tilde{W}}_{k}(\omega)$ with $\hat{\tilde{W}}_{k}(\omega)=A_{k} /\left(s-\lambda_{k}\right)$ and $A_{k}=$ $N\left(\lambda_{k}\right) /\left[\mathrm{d} D\left(\lambda_{k}\right) / \mathrm{d} s\right]$. Since for real $R(\omega)$ and $X(\omega)$, $D(s)$ has only real coefficients, its zeros $\lambda_{k}$ are either real or complex conjugate pairs. Real $\lambda_{k}$ corresponds in the time-domain to a system of overly damped impulses:

$$
\tilde{W}_{k}(t)= \begin{cases}\tilde{W}_{k}^{+}(t)=A_{k} e^{\lambda_{k} t} H(t), & \lambda_{k}<0 \\ \tilde{W}_{k}^{-}(t)=-A_{k} e^{\lambda_{k} t} H(-t), & \lambda_{k} \geq 0\end{cases}
$$

causal if $\lambda_{k}<0$ and non-causal if $\lambda_{k} \geq 0$. For complex roots $\lambda_{k}=-\alpha+i \beta$, conjugate pair $\lambda_{k+1}=-\alpha-i \beta$ must exist to form:

$$
\begin{aligned}
\hat{\tilde{W}}_{(k, k+1)}(\omega) & \equiv \hat{\tilde{W}}_{k}(\omega)+\hat{\tilde{W}}_{k+1}(\omega) \\
& =\frac{A_{k}}{s-\lambda_{k}}+\frac{A_{k+1}}{s-\lambda_{k+1}}=\frac{B s+C}{(s+\alpha)^{2}+\beta^{2}},
\end{aligned}
$$

which corresponds to causal or non-causal reflection impulses depending on the sign of $\alpha$ :

$$
\begin{aligned}
& \tilde{W}_{(k, k+1)}(t) \\
& = \begin{cases}\tilde{W}_{(k, k+1)}^{+}(t)=e^{-\alpha t} H(t)\left[B \cos (\beta t)+\frac{C-\alpha B}{\beta} \sin (\beta t)\right], & \alpha \geq 0 \\
\tilde{W}_{(k, k+1)}^{-}(t)=-e^{-\alpha t} H(-t)\left[B \cos (\beta t)+\frac{C-\alpha B}{\beta} \sin (\beta t)\right], & \alpha<0 .\end{cases}
\end{aligned}
$$

Each pair can be physically regarded as the impedance

$$
Z=R_{0}+i\left(X_{1} \omega-X_{-1} / \omega\right)
$$

of a Helmholtz oscillator of resistance $R_{0}$, acoustic mass $X_{1}$, stiffness $X_{-1}$, damping rate $\alpha=\left(1+R_{0}\right) / 2 X_{1}$, damped and undamped resonant frequencies of $\beta=\sqrt{\omega_{0}^{2}-\alpha^{2}}$ and $\omega_{0}=\sqrt{X_{-1} / X_{1}}$, respectively.

The damped-harmonic-oscillator system of Eqs. (15) and (16) is more common among sound-absorbing 
materials than the purely damped system of Eq. (14). For harmonic applications, however, non-causal reflection $\tilde{W}^{-}(t)$, can be accommodated by the space-time equivalence of the incident wave, $u^{+}\left(x_{b}, t\right)=u^{+}\left(x_{b}-\right.$ $c^{+} \Delta t, t-\Delta t$ ), and conversion of Eq. (14) or Eq. (16) into its spatial equivalent:

$$
\begin{aligned}
u^{-}\left(x_{b}, t\right)= & -u^{+}\left(x_{b}, t\right)+\int_{0}^{\infty} \tilde{W}^{+}(\tau) u^{+}\left(x_{b}, t-\tau\right) \mathrm{d} \tau \\
& +\int_{-\infty}^{0} \tilde{W}^{-}(\tau) u^{+}\left(x_{b}+c^{+} \tau-c^{+} \Delta t, t-\Delta t\right) \mathrm{d} \tau
\end{aligned}
$$

recognizing that the future incident waves are spatially coming from upstream. Unconditionally stable implementation in the form of Eq. (9) can be devised regardless of temporal causality, and attainment of a harmonic state depends only on the persistence of the source and the stability of the implementation (Fung and $\mathrm{Ju}$ (2001)).

The accurate determination of causal, complex roots may however require better testing procedures than the values routinely obtained from, e.g. the impedance tube. Nonetheless, a conveniently chosen set of basis functions $1 /\left(s-\lambda_{k}\right)$ of causal $\lambda_{k}$ has proved effective in the representation of practical impedance data for timeaccurate computation of impulse reflection (Fung and Ju (2001)).

\section{MEAN FLOW EFFECTS}

The principal application of impedance is for the characterization and prediction of the absorption and reflection of waves. The surface on which impedance is defined needs not be the actual material interface for the immediate interest is not the detailed micro-mechanical processes but their resultant reflection measured at distances. The presence of a flow and its boundary layer over a sound-absorbing surface raises the questions of whether the acoustic properties are modified by the flow, and how and where these properties can be measured. Available techniques for impedance measurement have been limited to the measurement of acoustic properties under no flow conditions. We can at this point only address how the acoustics properties of a material measured under no flow condition can be used to predict the propagation of waves in a non-uniformly moving acoustic medium.

Assuming the wall acoustic properties are not modified by the flow, the presence of a flow over an impedance wall has the known effects of convection and refraction due to the variable flow and sound speeds across the boundary layer. How these effects are accounted for depends on the solution models used for the prediction. If the prediction is for the propagation and reflection of acoustic disturbances from a mean sheared flow with boundary conditions imposed on the mean no-slip-material-flow interface, then Eq. (1) is locally applicable and the corresponding TDIBC is straightforward. The resolution of wave propagation in the steep Mach number gradient of a turbulent boundary layer may become the issue to address but is beyond the scope of the present paper. Goldstein and Rice (1973) attempted the formulation of an effective impedance to account for the presence of a thin boundary layer in flow ducts, but the aim and validity of their approach and the construction of a practical impedance expression remain questionable.

If the boundary layer is thin compared with the wavelengths of interest and refraction is negligible, an incident plane wave of angle $\theta$ to the wall normal in a mean free stream of Mach number $M_{0}$ has the effective plane-wave impedance $Z^{\prime}$ (Ingard, 1959):

$$
Z^{\prime}=Z\left(1+M_{0} \sin \theta\right) \text {. }
$$

The implementation of Eq. (18) as TDIBC via Eq. (9) is straightforward and unconditionally stable, but the identification of the local incident angle $\theta$ and the choice of effective $M_{0}$ would be up to the matching between measured and predicted results. For unspecific wave structures, Myers (1980) proposed the convectionmodified impedance (with $-y$ the normal wall direction and $\hat{v}$ the $y$-velocity component), viz.

$$
\hat{v}=\hat{p} / Z+M_{0}(i \omega Z)^{-1} \partial \hat{p} / \partial x,
$$

to account for the local wave angle by taking the partial $x$-derivative tangent to wall on $\hat{p}$. Ju and Fung (2001) proposed the equivalent reflection-incidence relation

$$
\left\lfloor 1+M_{0} \hat{W}_{\mathrm{II}}(\omega) \frac{\partial}{\partial x}\right\rfloor \hat{v}^{-}=\left[\hat{W}(\omega)+M_{0} \hat{W}_{\mathrm{II}}(\omega) \frac{\partial}{\partial x}\right] \hat{v}^{+},
$$

where $\hat{v}^{ \pm}=\hat{v} \pm \hat{p}$ and $\hat{W}_{\text {II }}(\omega)=1 /[i \omega(1+Z)]$. The corresponding time-domain expression is

$$
\begin{aligned}
& v^{-}(t)+M_{0} \int_{-\infty}^{+\infty} W_{\mathrm{II}}(t-\tau) \frac{\partial}{\partial x} v^{-}(\tau) \mathrm{d} \tau \\
& =\int_{-\infty}^{+\infty}\left[W(t-\tau)+M_{0} W_{\mathrm{II}}(t-\tau) \frac{\partial}{\partial x}\right] v^{+}(\tau) \mathrm{d} \tau .
\end{aligned}
$$

For the impedance model of Eq. (17), $W(t)$ takes the form of Eq. (16), $W_{\mathrm{II}}(t)=\left(X_{1} \beta\right)^{-1} \sin (\beta t) e^{-\alpha t} H(t)$, and Eq. (20) assumes the recursive expression:

$$
v^{-}(t)=\Delta t S_{I}(t)+2 M_{0} \Delta t S_{\mathrm{II}}(t)-\left(\frac{\Delta t}{X_{1}}+1\right) v^{+}(t)
$$

where

$$
\begin{gathered}
S_{I}(t)=2 \cos (\beta \Delta t) e^{-\alpha \Delta t} S_{I}(t-\Delta t)-e^{-2 \alpha \Delta t} S_{I}(t-2 \Delta t) \\
+\frac{2}{X_{1}}\left\{v^{+}(t)-[\cos (\beta \Delta t)+\alpha \sin (\beta \Delta t) / \beta]\right. \\
\left.\times e^{-\alpha \Delta t} v^{+}(t-\Delta t)\right\},
\end{gathered}
$$


and

$$
\begin{aligned}
S_{\mathrm{II}}(t)= & 2 \cos (\beta \Delta t) e^{-\alpha \Delta t} S_{\mathrm{II}}(t-\Delta t)-e^{-2 \alpha \Delta t} S_{\mathrm{II}}(t-2 \Delta t) \\
& +\frac{1}{X_{1} \beta} \sin (\beta \Delta t) e^{-\alpha \Delta t} \frac{\partial}{\partial x} p(t-\Delta t) .
\end{aligned}
$$

Recent application of these methods and comparisons with experiment has shown that the effective planewave impedance Eq. (18) provides a satisfactory account of mean flow effects for walls with high absorption ( $\mathrm{Ju}$ and Fung, 2001), whereas the implementation of Eqs. (20) and (21) would encounter the amplification of spurious waves generated at impedance discontinuity due to the tangential derivative. Although this instability can be suppressed by numerical smoothing, the advantage of the added complexity from the doubtful plane-wave assumption is difficult to justify over the much simpler effective plane-wave impedance. For highly reflective walls in high-speed flows, refraction effects in a shear layer must be addressed, schemes with higher resolution employed, but above all well-designed experiments conducted to address issues of impedance definition and modeling under convective environments.

\section{CONCLUSIONS}

We have reviewed the progress made in the extension of the concept of impedance for implementation as TDIBC for the computation of waves. This extension reveals the importance of causality in the characterization and modeling of the reflection and absorption of waves at the bounding surface of a domain. It suggests the broadband characterization of an impedance surface by a set of Helmholtz oscillators, which can be appropriately, efficiently and causally implemented as a two-level timeadvancement boundary scheme. The boundary thus treated renders a causal space-time continuation of the wall-bound and domain-entering waves as they propagate within the interior domain, rather than as a mathematical constraint spatially imposed on a physical or artificial boundary. The widely accepted concept of impedance for the closure of frequency-space-boundaryvalue problems thus extended should be applicable for the closure of a large class of initial-boundary-value problems in mechanics.

Perhaps the conventionally characterized, discretely measured impedance and other impedance-like transfer functions in the frequency-domain may also benefit from their re-characterization in the time-domain as a receptivity to or macro manifestation of the underlying sub-wave-scale possibly non-linear system dynamics.

\section{Acknowledgements}

Financial support on PolyU Grant 5144/99E is gratefully acknowledged.

\section{References}

Beggs, J.H., Luebbers, R.J., Yee, K.S. and Kunz, K.S. (1992) "Finitedifference time-domain implementation of surface impedance boundary conditions", IEEE Transactions on Antennas and Propagation 40(1), 49-56.

Botteldooren, D. (1994) "Acoustical finite-difference time-domain simulation in a quasi-cartesian grid", Journal of Acoustical Society of America 95(5), 2313-2319.

Botteldooren, D. (1995) "Finite-difference time-domain simulation of low-frequency room acoustic problems", Journal of Acoustical Society of America 98(6), 3302-3308.

Davis, S. (1991) "Low-dispersion finite difference methods for acoustic waves in a pipe", Journal of Acoustical Society of America 90(5), 2775-2781.

Fung, K.-Y. and Ju, H.B. (2001) "Broadband time-domain impedance models", AIAA Journal 39(8), 1449-1454.

Fung, K.-Y. and TallaPragada, B. (1997) "Time-domain impedance boundary condition for waves", In: Sommerfeldt, S., ed., Proceedings of Noise-Con 97 (The Penn State University, University Park, PA), pp $103-108$.

Fung, K.-Y., Ju, H.B. and TallaPragada, B. (2000) "Impedance and its time-domain extensions", AIAA Journal 38(1), 30-38.

Goldstein, M.E. and Rice, E. (1973) "Effect of shear on duct wall impedance", Journal of Sound and Vibration 30(1), 79-84.

Ingard, U. (1959) "Influence of fluid motion past a plane boundary on sound reflection, absorption, and transmission", Journal of Acoustical Society of America 31, 1035-1036.

Ju, H.B. and Fung, K.-Y. (2000) "A time-domain method for duct acoustics", Journal of Sound and Vibration 237(4), 667-681.

$\mathrm{Ju}$, H.B. and Fung, K.-Y. (2001) "Time-domain impedance boundary conditions with mean flow effects", AIAA Journal 39(9), $1683-1690$

Ju, H.B. and Fung, K.-Y. (2002) "Time-domain simulation of acoustic sources over an impedance plane", Journal of Computational Acoustics 10(3), 311-329.

Lee, C.F., Shin, R.T. and Kong, J.A. (1992) "Time domain modeling of impedance boundary condition", IEEE Transactions on Microwave Theory and Techniques 40(9), 1847-1850.

Luebbers, R.J., Hunsberger, F.P., Kunz, K.S., Standler, R.B. and Schneider, M. (1990) "A frequency-dependent finitedifference time-domain formulation for dispersive materials", IEEE Transactions on Electromagnetic Compatibility 32(3), $222-227$.

Maloney, J.G. and Smith, G.S. (1990) "Implementation of surface impedance concepts in the finite difference time domain (FDTD) technique". Proc. 1990 IEEE Antennas Propagat. Soc. Int. Symp., Dallas, TX, 4, 1628-1631.

Maloney, J.G. and Smith, G.S. (1992) "The use of surface impedance concepts in the finite-difference time-domain method", IEEE Transactions on Antennas and Propagation 40(1), $38-48$.

Myers, M.K. (1980) "On the acoustic boundary condition in the presence of flow", Journal of Sound and Vibration 71(3), 429-434.

Oh, K.S. and Schutt-Aine, J.E. (1995) "An efficient implementation of surface impedance boundary conditions for the finite-difference timedomain method", IEEE Transactions on Antennas and Propagation 43(7), 660-666.

Özyörük, Y. and Long, L.N. (1997) "A time-domain implementation of surface acoustic impedance condition with and without flow", Journal of Computational Acoustics 5(3), 277-296.

Özyörük, Y. and Long, L.N. (2000) "Time-domain calculation of sound propagation in lined ducts with sheared flows", AIAA Journal 38(5), $768-773$.

Özyörük, Y., Long, L.N. and Jones, M.G. (1998) "Time-domain numerical simulation of a flow-impedance tube", Journal of Computational Physics 146(1), 29-57.

Pierce, A.D. (1991) Acoustics (Acoustical Society of America, New York), pp 107-108.

Roden, J.A. and Gedney, S.D. (1999) "The efficient implementation of the surface impedance boundary condition in general curvilinear coordinates", IEEE Transactions on Microwave Theory and Techniques 47(10), 1954-1963.

Sullivan, D.M. (1992) "Frequency-dependent FDTD methods using z-transform", IEEE Transactions on Antennas and Propagation 40(10), 1223-1230. 
Tam, C.K.W. and Auriault, L. (1996) "Time-domain impedance boundary conditions for computational acoustics", AIAA Journal 34(5), 917-923.

Yee, K.S. and Chen, J.S. (1997) "Impedance boundary condition simulation in the FDTD/FVTD hybrid", IEEE Transactions on Antennas and Propagation 45(6), 921-925.

Yee, K.S., Shlager, K. and Chang, A.H. (1992) "An algorithm to implement a surface impedance boundary condition for
FDTD”, IEEE Transactions on Antennas and Propagation 40(7), $833-837$.

Zheng, S. and Zhuang, M. (2002). "Application and verification of time domain impedance boundary conditions in multi-dimensional acoustic problems." AIAA 2002-2593, 8th AIAA/CEAS Aeroacoustics Conference \& Exhibit, 17-19 June. 